\title{
How I Do It
}

Bertram Ng, MBBS Hong Kong, China ngbertram@yahoo.com.hk

This article is not about which is a better technique-strip or FUE. It is about accepting their advantages and limitations, and finding a way to make the best use of both.

I used to offer FUT to improve on previous FUE transplant, but was frustrated by donor depletion. A 31-year-old Chinese gentleman had 800 grafts extracted by manual FUE before. The donor density was down to $48 \mathrm{FU}$ per square centimeter. My strip yielded 2,056 grafts, or 3,192 hairs, giving a hair graft ratio of 1.55 . There were only 493 -hair FUs (2.4\%). I cannot imagine what could happen after automatic FUE

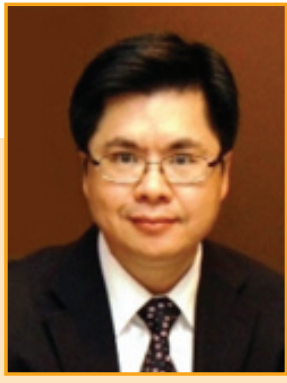
becomes a trend. This paper shows us how to keep some virgin soil for subsequent farming.

\section{Untouched strip: FUE combined with strip surgery to improve the FU number harvested in one session, preserving an untouched area for a possible future transplant}

\author{
Márcio Crisóstomo, MD Fortaleza, Ceará, Brazil marcio@implantecapilar.med.br
}

\section{Introduction}

The follicular unit transplant achieves very good and natural results. In order to avoid a linear scar in the donor area and increase its potential size, follicular unit extraction (FUE) is an alternative technique to strip harvesting $(\mathrm{SH}) .^{1-3}$ As FUE requires a high level of artisanship and is much more laborious than $\mathrm{SH}$, in the same surgical time a smaller number of follicular units (FUs) is harvested with FUE, even with powered FUE. ${ }^{4,5}$ Many surgeons resist FUE due to its long learning curve, technical difficulty, and higher rates of follicle transection, and because it gives fewer FUs than $\mathrm{SH}^{4}$ and patients usually want more hair, especially in an era of mega and gigasessions with three to more than five thousand FUs transplanted in one session. ${ }^{6-8}$

In more advanced cases of baldness (e.g., Norwood class $\mathrm{VI}$ ), the patient and doctor know that usually a second or even a third surgical procedure will be necessary to achieve a good density in the larger area of alopecia (Figure 1).

In order to improve the number of FUs transplanted, especially in larger bald areas or in cases of poor donor area, a combination of SH and FUE can be done. ${ }^{9}$ When this combined harvest is done, subsequent procedures that encounter the area where the FUE was done with the first procedure will have 20\%-40\% fewer FUs and many small punctiform scars. This adds local fibrosis after healing, which can make slivering and FU preparation difficult in

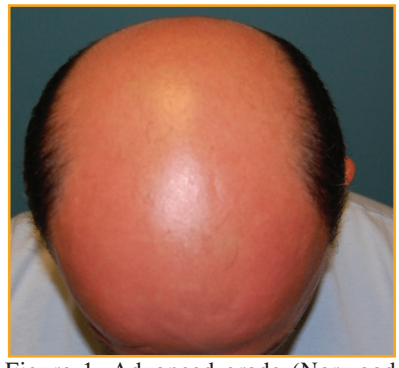

Figure 1. Advanced grade (Norwood VI) of baldness in which more than one surgery is needed.

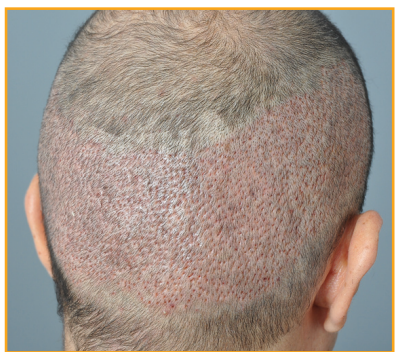

Figure 2. FUE sites on the third postoperative day.

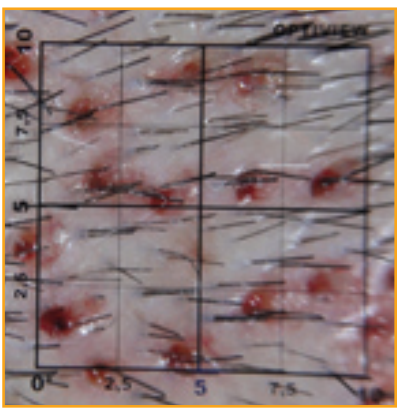

Figure $3.1 \mathrm{~cm}^{2}$ close-up of FUE showing $25 \%$ density decrease (11/44). the subsequent SHs. ${ }^{10}$ It can also change the natural anatomy and orientation of the FUs and make a new FUE session in this area more difficult and less productive (Figures 2, 3, and 4). ${ }^{2,10}$ For these reasons, the number of FU grafts harvested in a second procedure being performed in a previous FUE area can be decreased.

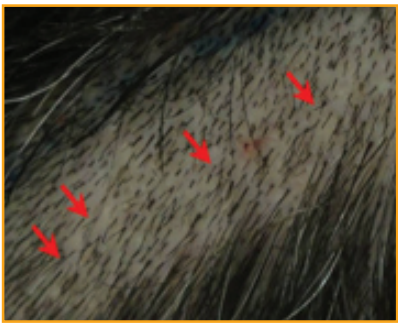

Figure 4. Minimal scars of FUE decreasing density and adding fibrosis in the donor area.

The aim of this work is to emphasize the importance of preserving an untouched area with its natural characteristics for the $\mathrm{SH}$ of a patient's possible next procedure.

\section{Surgical Technique: Untouched Strip}

First the safe donor area (SDA) is marked, where the hair will not fall with the baldness evolution (Figure 5), ${ }^{2,11}$ The strip for a megasession is excised and sutured in two layers, one deep with absorbable stitches of Monocry $\mathrm{l}^{\circledR} 3.0$ and a superficial running suture of Mononylon ${ }^{\circledR}$ 5.0. It is important that no undermining is done. Soon after, the FUE is harvested with sharp punches of $0.9 \mathrm{~mm}$ or $1.0 \mathrm{~mm}$, inside the SDA.

In patients with Norwood class V and VI, and some patients class VII, the author suggests that the surgeon leave an area of $1-1.5 \mathrm{~cm}$ just below the suture of the first strip without harvesting grafts by FUE (Figures 6 and 7). ${ }^{12}$ This will preserve an untouched area for a possible future transplant, which is called Untouched Strip, and it will keep its features

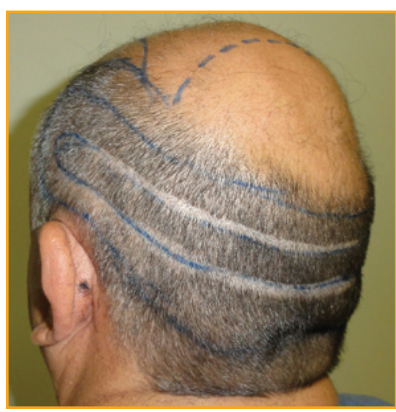

Figure 5. Demarcation of the safe donor area and the strip for a megasession.

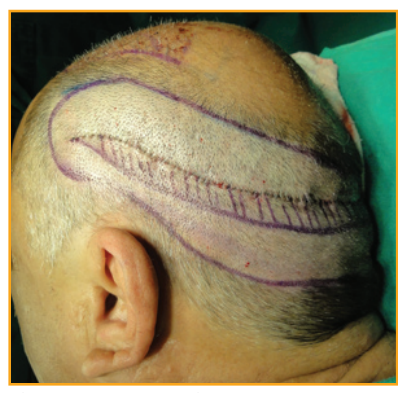

Figure 6. Demarcation of the safe donor area and the Untouched Strip below the suture of the first strip. 
of FU density and avoid the fibrosis and related anatomic distortion caused by the FUE scars (Figure 8). ${ }^{10,12}$

The FU implantation in the bald area is then done according to the surgeon's preference.

\section{Discussion}

One of the principles credited to Hippocrates in the Corpus Hippocraticus (Epidemics) is, "The physician must...have two special objects in view with regard to disease, namely, to do good or to do no harm." This is the principle of the Untouched Strip: to do no harm to an important area to be used in a possible future procedure (Figure 9).

The author uses the combination of FUE and SH in patients with more advanced baldness grades and/or in patients with poor donor area. ${ }^{9}$ It is very important to take care to harvest the FUE only in the SDA described by Unger, to avoid extracting hairs the patient could lose during his lifetime. ${ }^{2,13}$ To avoid this longterm drawback, the author has three caveats:

1. A careful analysis of

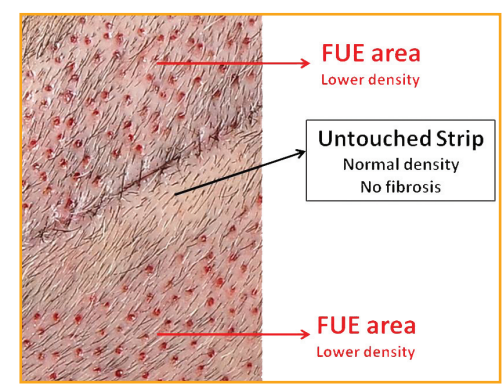

Figure 9. Close-up view showing the decrease of FU density in FUE areas and the Untouched Strip that keeps its normal density and will not have fibrosis. the family history to assess the patient's likelihood of developing a Norwood class VII should be done.

2. Older patients are preferable, usually older than 40 years old. Even though baldness undoubtedly evolves, only $16 \%$ of male patients will have Norwood class VII at the age of $80 .^{2}$

3. Most importantly, only patients with well-established baldness grades, especially in the crown area, normally Norwood class V and VI, and some cases of class VII (Figures 1 and 5 ), are candidates for the most aggressively defined SDA.

Exceptional cases are studied very carefully. It is important to avoid harvesting outside the SDA, but it is equally difficult to determine the real SDA without underestimating the patient's real potential donor area.

In larger cases, even with the combined technique, a second procedure may be needed. In these cases, to avoid the decrease of FU density in the donor area and the fibrosis that can compromise the second procedure, ${ }^{10}$ the Untouched Strip is done. The Untouched Strip can be marked below or above the strip suture. Although Unger and Cole prefer to harvest from the inferior toward the superior part in the donor area,${ }^{2}$ the author prefers to leave the untouched area below the suture (Figure 8). This leaves the superior portion for FUE, which is usually easier and more productive in this region.

In the second surgery, normally done 8-12 months later, the time necessary for the donor area to recover its elasticity, the surgeon will perform another FUE session or, more commonly, $\mathrm{SH}$ with or without the excision of the previous scar. This second procedure is a personal preference of the surgeon that should be discussed with the patient. The key point is that the Untouched Strip keeps its natural features for the next chosen procedure (Figure 9). ${ }^{12}$

Tsilosani prefers to perform FUE first when using the combined procedure ${ }^{9}$ but, because the FUs obtained with FUE are usually more skinny and thus more susceptible to ischemia-reperfusion injury, ${ }^{14}$ this author's routine is to do the FUE later to reduce the time FUs will be outside the body. ${ }^{12}$

One advantage of the combined procedure is the possibility of decreased tension on the wound closure after performing the FUE leading to a better scar quality. ${ }^{9}$

The main advantage of this combined technique is the increased number of FUs transplanted in one procedure. Tsilosani published an increase of $14 \%-42 \%$ with the combined surgery, with an average of $29.5 \% .^{9}$ This is very desirable, especially in patients with poor donor area in terms of density or elasticity and/or with large bald areas. In the Untouched Strip, this increase is slightly lower because there is no FUE harvesting in the preserved area, but the benefit of not having scar tissue and having normal or almost normal FU density and less hair follicle anatomy distortion in this area compensates for this in a future procedure. ${ }^{12}$

\section{Conclusion}

The combined use of FUE and SH can expand the graft number in one session, thus providing a better density and coverage in the first surgery. The Untouched Strip protects the donor area for a future

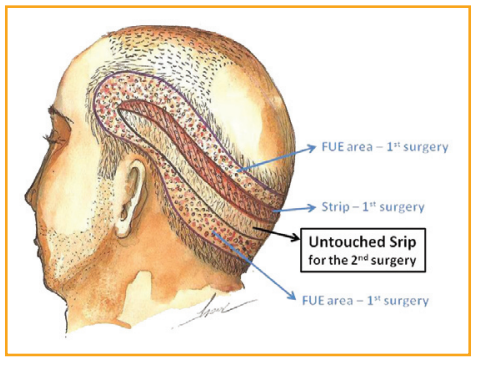

Figure 10. The FUE area (inside the safe donor area) above and below the strip for the first surgery, leaving $1.0-1.5 \mathrm{~cm}$ of an Untouched Strip preserving the donor area for a possible future transplant. second procedure that will not be damaged by FUE scarring or decreased FU density (Figure 10).

\section{References}

1. Rassman, W.R., et al. Follicular unit extraction: minimally invasive surgery for hair transplantation. Dermatol Surg. 2002; 28(8):720-728.

2. Unger, W.P., and J. Cole. Donor Harvesting. In: W.P. Unger and R. Shapiro. Hair Transplantation. 4th Ed. New York: Marcel Dekker. 2004; pp. 301-348.

3. Harris, J.A. Follicular Unit Extraction. In: M.R. Avram and N.E. Rogers. Hair Transplantation. New York: Cambridge University Press, 2010; pp. 23-34.

4. Dua, A., and K. Dua. Follicular unit extraction hair transplant. J Cutan Aesth Surg. 2010(May-Aug); 3(2):76-81.

5. Harris, J.A. New methodology and instrumentation for follicular unit extraction: lower follicle transection rates and expanded patient candidacy. Dermatol Surg. 2006(Jan); 32(1):56-61.

6. Seager, D.J. The "one-pass hair transplant"- a six year perspective. Hair Transplant Forum Int'l. 2002; 12(5):1-6. 
7. Crisóstomo, M.R. Gigasessions-larger sessions for baldness grades IV to VI. Presented at the XIII International Congress of Italian Society for Hair Restoration. Capri, Italy; May 2010.

8. Wong, J. Preoperative Care for Super Mega-Sessions. In: D. Pathomvanich and K. Imagawa. Hair Restoration Surgery in Asians. Springer. 2010; pp. 81-82.

9. Tsilosani, A. Expanding graft numbers combining strip and FUE in the same session: effect on linear wound closure forces. Hair Transplant Forum Int'l. 2010; 20(4):121-123.

10. Bernstein, R.M, W.R. Rassman, and K.W. Anderson. FUE megasessions - evolution of a technique. Hair Transplant Forum Int'l. 2004; 14(3):97-99.

11. Jimenez, F. The Donor Area. In: M.R. Avram and N.E. Rogers. Hair Transplantation. New York: Cambridge University Press. 2010; pp. 15-21.

12. Crisóstomo, M.R. Aplicação do follicular unit extraction no transplante capilar. Presented at the 30th Jornada Carioca de Cirurgia Plástica of the Brazilian Society of Plastic Surgery. Rio de Janeiro, Brazil; August 2011.

13. Unger, W. Letters to the Editors. Re: Combining Strip and FUE. Hair Transplant Forum Int'l. 2010; 20(5):170.

14. Crisóstomo, M.R., et al. Oxidative stress in follicular units during hair transplantation surgery. Aesthetic Plast Surg. 2011(Feb); 35(1):19-23.

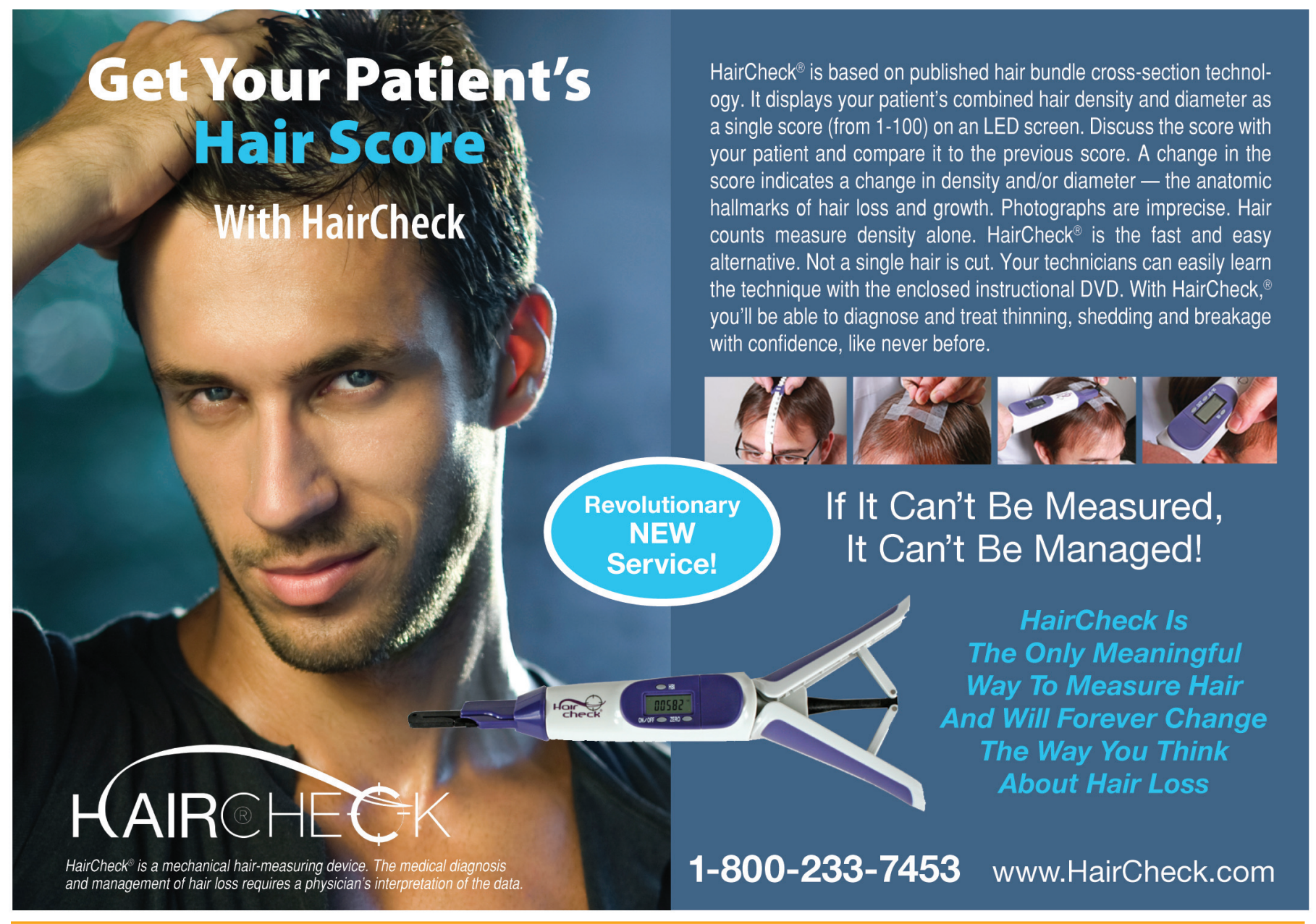

